\title{
(Q) Neurodevelopment and ages of onset in depressive disorders
}

\author{
Cindy C Hagan, Julia M E Graham, Paul O Wilkinson, Nick Midgley, John Suckling, Barbara J Sahakian, Ian M Goodyer
}

Lancet Psychiatry 2015; 2: 1112-16

Department of Psychiatry (CC Hagan PhD, J M E Graham PhD, $P O$ Wilkinson $M D$, Prof J Suckling PhD, Prof BJ Sahakian DSC, Prof I M Goodyer MD), Medical Research Council and Wellcome Trust Behavioural and Clinical Neuroscience Institute (Prof) Suckling, Prof BJ Sahakian), and Wellcome Trust Neurosciences in Psychiatry Network (Prof I M Goodyer, Prof J Suckling), University of Cambridge, Cambridge, UK; Department of Psychology, Columbia University, New York, NY, USA (C C Hagan); and Anna Freud Centre and Research Department of Clinical, Educational and Health Psychology, University College London, London, UK (N Midgley PhD)

Correspondence to: Dr Cindy C Hagan, Department of Psychology, Schermerhorn Hall, Columbia University, New York, NY 10027, USA cindy.hagan@gmail.com

How and why do clinical depressive disorders emerge in adolescence? In this Personal View, we present a neurodevelopmental theory to address causes for adolescent onsets of clinical depressive disorders. We argue that theories should account for three perplexing aspects of depressive disorders in adolescence: the episodic nature of depression; differences between sexes in rates of depression across development; and age-differentiated onsets. We consider how theories such as psychosocial acceleration, heterochronic brain development, dual-process models, glucocorticoid vulnerability hypothesis linked to early life stress, and epigenetic and genetic susceptibility might explain some aspects of adolescent depressive disorders. We argue that some synthesis between existing theories might be needed to establish a sufficient neurodevelopmental theoretical framework to explain onsets of depressive disorders in adolescence.

\section{Introduction}

About half of all adult mental illnesses emerge in adolescence, ${ }^{1}$ with clinical depressive disorders contributing the greatest incidence risk, but little is known as to how or why this pattern occurs. Different theories have attempted to explain this early emergence, including accelerated maturation of sex hormones, ${ }^{2}$ aberrant brain development, ${ }^{3-6}$ abnormal responses to life stress, ${ }^{7,8}$ and differences in genetic and environmental susceptibility ${ }^{9-1}$ (figure). However, no single theory alone provides a satisfactory neurodevelopmental answer.

In view of the perplexing manifestations of depressive illness, such as episodic occurrences, ${ }^{12}$ a female preponderance, ${ }^{13,14}$ and adolescent onsets, ${ }^{1,11,13,14}$ might more than one of these theories contribute to the explanation of the developmental onset of depressive disorders in adolescence? We argue that a hybrid of these theories is might be necessary to explain the developmental emergence of depressive illnesses.

\section{Psychosocial acceleration}

Adolescence is a period of rapid brain and psychosocial development. Psychosocial acceleration theory suggests that the timing of hormonal maturation varies as a function of an individual's responsiveness to their rearing environment (via genetic or epigenetic mechanisms) and rearing environmental stability. Findings confirm that early and later pubertal timing are associated with various poor health outcomes in adult life, including depression. ${ }^{15}$ Unstable environmental rearing conditions are associated with greater unpredictability and uncertainty than are stable conditions, and might therefore induce stress in an individual, with behaviours such as indecisiveness and over-reactivity generalised to subsequently encountered environments. Individuals have to adapt more often in unstable environments than in stable environments, and such instability has been suggested to accelerate maturation in some individuals, but also render them susceptible to the development of mental illnesses, such as depression. ${ }^{16} \mathrm{~A}$ positive association has been reported between pubertal timing and self-reported depression in a large population sample of adolescents, ${ }^{17}$ but pubertal acceleration alone does not explain how the 1:1 incidence ratio of female to male depression in childhood becomes 2:1 in adolescence and adulthood. ${ }^{18}$ Gender socialisation might account for sex differences in rates of mental illness ${ }^{19}$ and neurodevelopment. For example, in instances of domestic abuse in which an individual has little control, female adolescents might favour socialising with delinquent peers over spending time at home more often than would male adolescents. ${ }^{19}$ Repeatedly experienced throughout development, these socialisation differences might partly account for neurodevelopmental sex differences. ${ }^{20}$ Additionally, changes in the primary caregiver, parental absence, or the introduction of a step-parent are examples of rearing environment changes that might also partly explain sex-related and age-related effects of mental illness. Considered together, sex might independently and interactively affect the developing brain ${ }^{21}$ and increase the proneness of some individuals to develop depression in adolescence.

\section{Heterochronic brain development}

Human brain development is heterochronic, meaning that different developmental timings and rates of change are shown across regions. ${ }^{22}$ Some researchers propose that atypical brain maturation, in combination with psychosocial, environmental, and biological factors, predisposes adolescents to different forms of psychopathological changes, including major depressive disorder. ${ }^{3}$ More recently, neuroimaging analysis techniques have been applied that concentrate on the coordinated development of neural systems distributed across proximal and distal brain regions. ${ }^{23}$ When applied to the aberrant brain maturation hypothesis, ${ }^{3}$ disparities in the timing or rate of synapse formation within neural systems, in combination with psychosocial, environmental, and biological factors, might confer an increased susceptibility to onset of mental illness, with depression being particularly likely in adolescence and early adulthood.

\section{Dual-process models}

Compatible with the heterochronic development of neural systems, dual-process models propose that so-called hot and cold systems underlie both typical and 
atypical behaviour in human beings. ${ }^{4-6}$ Hot systems were initially proposed to underlie emotionality and cold systems to underlie cognition with their interplay comprising a skillset used during the exertion of willpower. ${ }^{5}$ In brief, hot systems refer to bottom-up emotional processing arising in lower brain regions, passing upwards through the limbic-system structures and emerging as reactive or reflexive behaviours. Cold systems refer to top-down cognitive processing arising from frontal and prefrontal regions of the brain passing down towards the limbic system and emerging as proactive or reflective behaviours. The balance between hot and cold systems is driven by a set of factors, including developmental level, personal history, stress, and temperament, and can be further modulated by pharmacological intervention. ${ }^{5,6} \mathrm{~A}$ developmental imbalance between hot and cold systems is present in adolescence, whereby a mature hot system occurs alongside a relatively immature cold system. ${ }^{1,4}$ For example, the hot amygdala - a limbic region associated with the perception of biologically relevant socioemotional information ${ }^{24}$-matures earlier than does the cold hippocampus, implicated in memory, and the cold prefrontal cortex, implicated in the cognitive regulation of socioemotional stimuli., ${ }^{5,625-27}$ Some researchers therefore suggest that exaggerated neurodevelopmental imbalances between the reflexive hot system and the reflective cold system contribute to the emergence of affective disorders in adolescence. ${ }^{4}$ Caution should be taken when hot and cold nomenclature is assigned to neuropsychological tasks, however, because individuals with depressive disorders might have an increased sensitivity to feedback on task performance, thereby instilling negative expectations and a reinterpretation of a cold cognitive task as a hot emotional task. ${ }^{6}$ Unfortunately, cold-turned-hot thinking patterns are difficult to change, even with medication or targeted brain stimulation techniques. ${ }^{6}$ Repeated cold-turned-hot experiences in addition to early life stressors, such as rearing environment instability or chronic psychosocial difficulties, could promote accelerated development of the hot system via prompting of over activity and strengthening of connections between hot regions. This change can leave adolescents with depression bewildered about their fluctuating mood states. As one 15-year-old with clinical depression and a history of chronic trauma said: "It's not really easy to make sense of [because] when you're in that mood, you don't think of anything like you don't think logically, but then like once you've sort of calmed down and everything, I sort of sit and think 'Why was I like that?' [...] And it doesn't really make sense". ${ }^{28}$ Albeit in a small sample of 30 adolescents with depression, a prospective longitudinal neuroimaging study showed developmental acceleration of the hot amygdala, but only in adolescent girls with depressive disorders in comparison to healthy adolescents. ${ }^{20}$ Notably, adolescent boys with depressive disorders

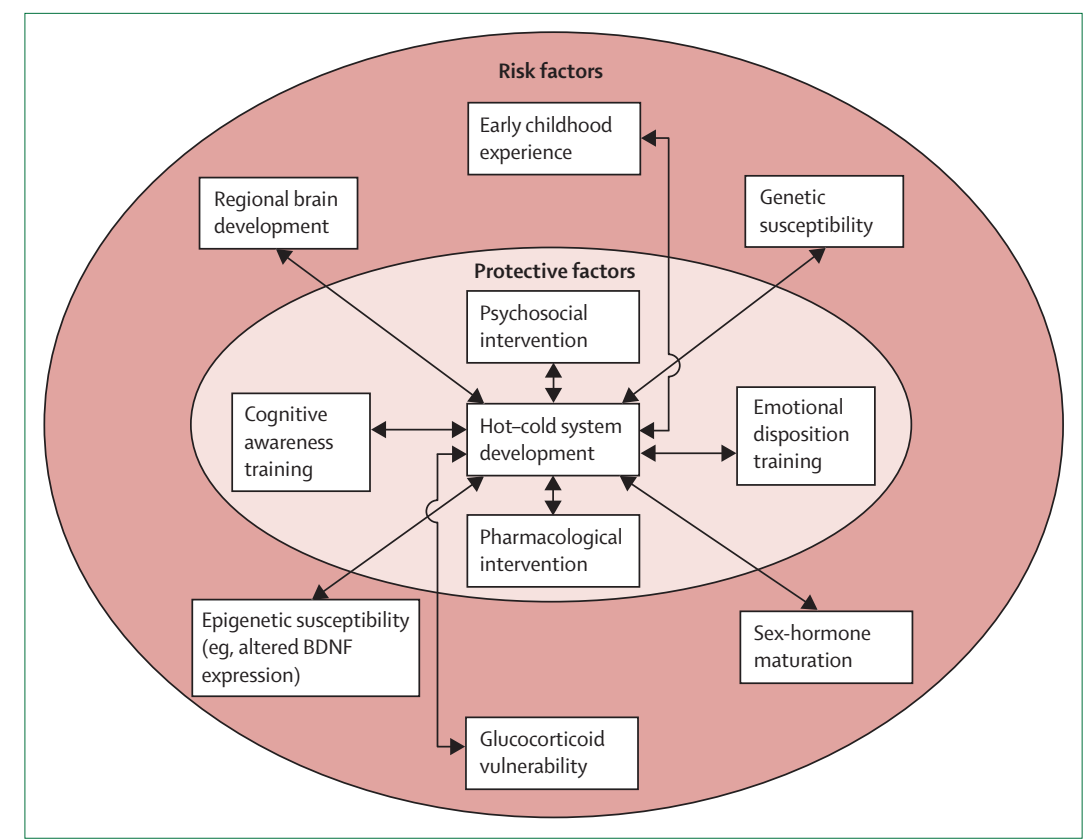

Figure: Risk and protective factors interacting with the development of hot and cold neural systems and associated with the development of depressive illness during adolescence $\mathrm{BDNF}=$ Brain-derived neurotrophic factor.

showed developmental attenuation of the amygdala. ${ }^{20}$ This finding suggests that pathways to depressive disorders in adolescence could be sex-specific.

Could attenuated development of the cold system also generate an exaggerated imbalance between hot and cold systems therefore conferring risk for major depressive disorder? Reductions in hippocampal volume have been reported in women with depression and a history of abuse in childhood compared with either women with depression and no history of childhood abuse or healthy women. ${ }^{29}$ Additionally, a large metaanalysis $^{30}$ of 15 studies comprising 1728 adult patients with depression and 7199 healthy adults showed significantly reduced hippocampal volume in individuals of both sexes who had become depressed at a young age ( $<21$ years), but only for those with recurrent episodes. Results in adolescents with depression are less clear, with cross-sectional studies showing both significant reductions and no significant differences in hippocampal volumes relative to healthy controls. ${ }^{31,32}$ Some investigators posit that the delayed developmental trajectory of the hippocampus compared with the amygdala hinders the detection of hippocampal volumetric changes until adulthood. ${ }^{27}$ Longitudinal studies account for variance within and across individuals and so are better suited than cross-sectional studies, which only assess variance across individuals, to detect differences in the developmental trajectories of brain regions, such as the hippocampus during adolescence. Other investigators have speculated that exposure to environmental stressors might initially potentiate, but with increasing chronicity or severity 
eventually attenuate, development of the cold system in some individuals, leading to reduced neural connectivity between brain regions and protracted overall growth. ${ }^{5}$ This view is harmonious with the suggestion that dysfunctional cold cognitive processes precede depressive illness $\mathrm{s}^{6,25}$ and with prospective longitudinal data associating attenuated hippocampal growth in adolescence with development of depression in male and female adolescents. ${ }^{20}$

\section{Glucocorticoid vulnerability}

Attenuated development of the cold system is compatible with the glucocorticoid vulnerability hypothesis, which suggests that chronic stress negatively interferes with people's ability to withstand and overcome trauma-related neuropathological changes. ${ }^{7}$ Some researchers propose that brain structural and functional malleability (ie, neuroplasticity) is perturbed by chronic stress or stress dysregulation, contributing to the emergence of depressions. $^{8}$ Treatment with antidepressant drugs inhibits and sometimes reverses neuroplastic damage, particularly in the hippocampus. ${ }^{8}$ The hippocampus, a core region of the cold system, is particularly susceptible to atrophy, perhaps resulting from epigenetic effects on BDNF (a trophic protein important for neurodevelopment) gene expression ${ }^{9}$ or, in view of the abundance of glucocorticoid (specifically cortisol in human beings) receptors in the hippocampus, from toxic concentrations of corticoids circulating in the brain (ie, acquired neuroendangerment). ${ }^{25}$ Notably, patients with Cushing's disease, which is associated with hippocampal atrophy and hypercortisolaemia resulting from the increased production and secretion of glucocorticoids, often have a depressive illness. Experiments in rodents ${ }^{7}$ show that stress can downregulate glucocorticoid receptors in the hippocampus, leading to an overabundance of glucocorticoids. The hippocampus typically exerts an inhibitory effect on the stress response. However, in conditions of chronic stress, the inhibitory response by the hippocampus is impaired. ${ }^{7}$ In line with these findings in rodents, high concentrations of evening cortisol and symptoms of post-traumatic stress disorder have been related to volumetric reductions in the hippocampus of children with a history of maltreatment. ${ }^{33}$ Immediate and long-term effects of cortisol hypersecretion on the hippocampus probably depend on the developmental stage of the hippocampus at the time of stress exposure,,$^{27,33,34}$ the amount and chronicity of cortisol release induced by the stressor, ${ }^{27,33}$ and the severity and chronicity of stress exposure. ${ }^{27,33}$ Accordingly, an epidemiological study ${ }^{35}$ reported a fourfold increase in risk of childhood memory impairments in adults who had been exposed to more than three adverse experiences during childhood or adolescence. By contrast, acute stress in adulthood might lead to reparable hippocampal damage. ${ }^{7}$ Aberrant hippocampal function could contribute to impaired concentration, anhedonia, or stress dysregulation through connectivity with the dorsolateral prefrontal cortex, nucleus accumbens, and hypothalamus. Individuals with depression might be more susceptible to corticoid-mediated acquired neuroendangerment of the hippocampus, which could therefore have downstream effects on experience-dependent learning. ${ }^{25}$ Hence, depressive episodes could emerge in vulnerable adolescents when stressful life events occur and trigger depressive symptoms..$^{25}$ A study has shown that many adolescents with clinical depression link the occurrence of depressive episodes to stressful life events, leaving them unable to use the reflective cold system to regulate an (over)active hot system (Midgley $\mathrm{N}$ and colleagues, unpublished). A 16-year-old girl with severe depression who had been exposed to severe domestic violence between her parents explained that: “There's loads and loads of things that are flying around in my head and I can't stop them and look at them and find out what exactly it is and what caused them, I just know that like when it happens it makes you feel sick and dizzy and just horrible" (Midgley N and colleagues, unpublished). ${ }^{28}$

\section{Epigenetic and genetic susceptibility}

Changes in BDNF gene expression is one mechanism to explain sex-differentiated risk for depression as a function of early life experience. Because biological sex is associated with different rates of development in different brain regions, ${ }^{21}$ epigenetic regulation of gene expression in the brain might occur in both a sex-specific and age-specific way. ${ }^{9,10}$ Accordingly, female mice repeatedly bitten by a larger, more aggressive mouse of the same sex showed amplified social withdrawal behaviour and increased BDNF protein expression in the bed nucleus of the stria terminalis, ${ }^{36}$ a region of the brain associated with threat vigilance. ${ }^{37}$ Male mice do not show these same effects. ${ }^{36}$ Downregulated BDNF gene expression in the mouse hippocampus, induced by prenatal toxin exposure, has been associated with changes in brain function and behaviour.' In human beings, peripheral blood concentrations of BDNF protein might indicate the state of depressive illness. ${ }^{38}$ Although similar concentrations of BDNF protein have been reported in both healthy adults and adults in full remission of depression, lower concentrations have been recorded in patients with current depression..$^{38}$ A separate study ${ }^{9}$ showed that prenatal exposure to environmental toxins leads to sex-specific changes in DNA methylation of $B D N F$ that are associated with sex-specific effects on behaviour in both mice and human beings. Hypemethylation reduces gene expression of hippocampal $B D N F$ and could therefore function as a biomarker for exposure to adversity in early life, leading to sexdifferentiated patterns of behaviour in later life. ${ }^{9}$ Could sex-specific methylation patterns of hippocampal $B D N F$ partly explain sex and age differences in rates of depressive illness? 
Although little is known about how gene-environment associations affect human neurodevelopment and onsets of depressive illness, ${ }^{39}$ some evidence suggests that genes help to modulate adolescent development and might accordingly change the risk for the emergence of depression in the second decade of life (aged 10-19 years) compared with the first (aged 0-9 years). One longitudinal twin study ${ }^{11}$ has shown that differential genetic effects are greater for the onset of depression in adolescence than for that in childhood, suggesting an additional pathway for sex and age differentiation of depressive disorders because such genes might also affect adolescent brain development. These gene-gene and gene-environment relations are yet to be fully clarified and incorporated into a neurodevelopmental framework.

\section{Conclusion}

To summarise, synthesis between existing theories is needed to provide a sufficient neurodevelopmental theoretical framework with which to explain adolescent onsets of clinical depressions. Although alternative explanations cannot be ruled out, risk mechanisms might be differentially impaired among different individuals, giving rise to the clinical heterogeneity often reported in depressive disorders (figure). Similarly, different amounts and combinations of intervention techniques might be necessary for treatment of individuals with different clinical profiles of depression (figure). Nevertheless, irrespective of the clinical manifestation of depression, intervention at the earliest stages of illness onset might be important to prevent the neural and cognitive imprints that fuel recurrence risks into adult life..$^{40}$ To assist in the early detection and treatment of depression, clinicians working with parents, who themselves might be mentally unwell, could improve the education of patients about the environmental and physical risk factors associated with development of major depressive disorder, the symptoms of this disorder in adolescence, and could enable discussions to improve parent-child communication and interaction. Perhaps therapeutic studies of adolescents with depression and either a positive family history of major depressive disorder or early exposure to suboptimum environments could consider trialling treatment with DNA demethylases (thus normalising gene expression) or histone deacetylase inhibitors (which disrupt DNA translation) in an effort to mitigate epigenetic manifestations of the disorder. ${ }^{41}$ Furthermore, public health campaigns and educational establishments could ensure exposure of all children to enriched educational environments, assist in educating the general public (including adolescents) about the symptoms associated with major depressive disorder in adolescence, and disseminate knowledge about the risks associated with individuals who have early sexual maturation and development. Finally, in addition to the therapeutic treatment of adolescents, perhaps training interventions could focus on instilling positive thinking patterns and self-monitoring of cognitions to improve executive function skills. Stress regulation strategies could be developed and introduced as part of personal growth and education in schools in an effort to cultivate the growth of cold system regions and reduce the reliance on behaviours attributable to the hot system. ${ }^{4}$ As the parent of an adolescent with depression explained after a short-term therapeutic intervention that focused on helping adolescents to develop a greater balance between the reflective cold and the reflexive hot processing systems: "He's certainly not in the dark place where he was...he can now say 'this is upsetting me' or 'that makes me angry'-he's now able to analyse some of his feelings" (Midgley $\mathrm{N}$ and colleagues, unpublished). ${ }^{28}$ Early provision of treatment, be it psychological, pharmacological, or both, aimed to establish optimum balance between top-down cold and bottom-up hot cognitive processes, might be most effective for recovery from and relapse prevention of depression..$^{40,42}$

Contributors

$\mathrm{CCH}$ and IMG drafted the manuscript. All authors provided insightful comments, edited, and approved the final version of the manuscript.

\section{Declaration of interests}

IMG received personal fees from Lundbeck, outside the submitted work. BJS consults for Cambridge Cognition, Servier, Peak (Brainbow), Otsuka, and Lundbeck. BJS received a grant from Janssen/Kohnson \& Johnson, and has share options in Cambridge Cognition. POW has receieved personal fees from Lundbeck and Takeda, outside the submitted work, and is as a supervisor and trainer for interpersonal psychotherapy. All other authors declare no competing interests.

\section{Acknowledgments}

IMG is supported by a Wellcome Trust Strategic Award (grant 09584) and a NIHR HTA Trials Award (grant 006/474, ISRCTN83033550). This work was supported by the UK Medical Research Council (grant G0802226). We thank the participants of the IMPACT-ME (My Experience) project, funded by the Monument Trust, for providing insight into the experience of depression in adolescence and the effects of treatment. We also thank Karl Mobbs and Frances Champagne for providing helpful comments on early drafts of this manuscript. This Personal View was written with the National Institute of Health Research Cambridge Biomedical Research Centre and the Neuroscience in Psychiatry Network.

\section{References}

1 Jones PB. Adult mental health disorders and their age at onset Br J Psychiatry Suppl 2013; 54: s5-10.

2 Belsky J, Steinberg L, Draper P. Childhood experience, interpersonal development, and reproductive strategy: and evolutionary theory of socialization. Child Dev 1991; 62: 647-70.

3 Paus T, Keshavan M, Giedd JN. Why do many psychiatric disorders emerge during adolescence? Nat Rev Neurosci 2008; 9: 947-57.

4 Casey BJ, Jones RM, Levita L, et al. The storm and stress of adolescence: insights from human imaging and mouse genetics. Dev Psychobiol 2010; 52: 225-35.

5 Metcalfe J, Mischel W. A hot/cool-system analysis of delay of gratification: dynamics of willpower. Psychol Rev 1999; 106: 3-19.

6 Roiser JP, Sahakian BJ. Hot and cold cognition in depression. CNS Spectr 2013; 18: 139-49.

7 Sapolsky RM, Krey LC, McEwen BS. The neuroendocrinology of stress and aging: the glucocorticoid cascade hypothesis. Endocr Rev 1986; 7: 284-301.

8 Pittenger C, Duman RS. Stress, depression, and neuroplasticity: a convergence of mechanisms. Neuropsychopharmacology 2008; 33: 88-109.

9 Kundakovic M, Gudsnuk K, Herbstman JB, Tang D, Perera FP, Champagne FA. DNA methylation of $B D N F$ as a biomarker of earlylife adversity. Proc Natl Acad Sci USA 2014; 112: 201408355. 
10 Jessen HM, Auger AP. Sex differences in epigenetic mechanisms may underlie risk and resilience for mental health disorders. Epigenetics 2011; 6: 857-61.

11 Nivard MG, Dolan CV, Kendler KS, et al. Stability in symptoms of anxiety and depression as a function of genotype and environment: a longitudinal age study from 3 to 63 years. Psychol Med 2015; 45: 1039-49.

12 National Institute of Mental Health. Depression. 2011. http://www. nimh.nih.gov/health/publications/depression/depressionbooklet_34625.pdf (accessed July 24, 2015)

13 Kessler RC, Berglund P, Demler O, Jin R, Merikangas KR, Walters EE. Lifetime prevalence and age-of-onset distributions of DSM-IV disorders in the National Comorbidity Survey Replication. Arch Gen Psychiatry 2005; 62: 593-602.

14 Kessler RC, Wang PS. The descriptive epidemiology of commonly occurring mental disorders in the United States. Annu Rev Public Health 2008; 29: 115-29.

15 Day FR, Elks CE, Murray A, Ong KK, Perry JRB. Puberty timing associated with diabetes, cardiovascular disease and also diverse health outcomes in men and women: the UK Biobank study. Sci Rep 2015; 5: 11208.

16 Graber JA. Pubertal timing and the development of psychopathology in adolescence and beyond. Horm Behav 2013; 64: 262-69.

17 Kaltiala-Heino R, Kosunen E, Rimpelä M. Pubertal timing, sexual behaviour and self-reported depression in middle adolescence. J Adolesc 2003; 26: 531-45.

18 Maughan B, Collishaw S, Stringaris A. Depression in childhood and adolescence. J Can Acad Child Adolesc Psychiatry 2013; 22: 35-40.

19 Whitney SD, Renner LM, Herrenkohl TI. Gender differences in risk and promotive classifications associated with adolescent delinquency. J Genet Psychol 2010; 171: 116-38.

20 Whittle S, Lichter R, Dennison M, et al. Structural brain development and depression onset during adolescence: a prospective longitudinal study. Am J Psychiatry 2014; 171: 564-71.

21 Goddings AL, Mills KL, Clasen LS, Giedd JN, Viner RM, Blakemore SJ. The influence of puberty on subcortical brain development. Neuroimage 2014; 88: 242-51.

22 Giedd JN, Blumenthal J, Jeffries NO, et al. Brain development during childhood and adolescence: a longitudinal MRI study. Nat Neurosci 1999; 2: 861-63.

23 Alexander-Bloch A, Giedd JN, Bullmore E. Imaging structural co-variance between human brain regions. Nat Rev Neurosci 2013; 14: 322-36.

24 Vrtička P, Sander D, Vuilleumier P. Lateralized interactive social content and valence processing within the human amygdala. Front Hum Neurosci 2012; 6: 358.

25 Goodyer IM. Emanuel Miller Lecture: early onset depressionsmeanings, mechanisms and processes. J Child Psychol Psychiatry 2008; 49: 1239-56.

26 Ochsner KN, Ray RR, Hughes B, et al. Bottom-up and top-down processes in emotion generation: common and distinct neural mechanisms. Psychol Sci 2009; 20: 1322-31.
27 Tottenham N, Sheridan MA. A review of adversity, the amygdala and the hippocampus: a consideration of developmental timing. Front Hum Neurosci 2009; 3: 68.

28 Midgley N, Ansaldo F, Target M. The meaningful assessment of therapy outcomes: incorporating a qualitative study into a randomized controlled trial evaluating the treatment of adolescent depression. Psychotherapy (Chic) 2014; 51: 128-37.

29 Vythilingam M, Heim C, Newport J, et al. Childhood trauma associated with smaller hippocampal volume in women with major depression. Am J Psychiatry 2002; 159: 2072-80.

30 Schmaal L, Veltman DJ, van Erp TGM, et al. Subcortical brain alterations in major depressive disorder: findings from the ENIGMA Major Depressive Disorder working group. Mol Psychiatry 2015; published online June 30. DOI:10.1038/mp.2015.69.

31 MacMaster FP, Kusumakar V. Hippocampal volume in early onset depression. BMC Med 2004; 2: 2.

32 Rosso IM, Cintron CM, Steingard RJ, Renshaw PF, Young AD, Yurgelun-Todd DA. Amygdala and hippocampus volumes in pediatric major depression. Biol Psychiatry 2005; 57: 21-26.

33 Carrion VG, Weems CF, Reiss AL. Stress predicts brain changes in children: a pilot longitudinal study on youth stress, posttraumatic stress disorder, and the hippocampus. Pediatrics 2007; 119: 509-16.

34 Romeo RD, McEwen BS. Stress and the adolescent brain. Ann N Y Acad Sci 2006; 1094: 202-14.

35 Anda RF, Felitti VJ, Bremner JD, et al. The enduring effects of abuse and related adverse experiences in childhood. A convergence of evidence from neurobiology and epidemiology. Eur Arch Psychiatry Clin Neurosci 2006; 256: 174-86.

36 Greenberg GD, Laman-Maharg A, Campi KL, et al. Sex differences in stress-induced social withdrawal: role of brain derived neurotrophic factor in the bed nucleus of the stria terminalis. Front Behav Neurosci 2014; 7: 223.

37 Davis M, Whalen PJ. The amygdala: vigilance and emotion. Mol Psychiatry 2001; 6: 13-34.

38 Molendijk ML, Bus BAA, Spinhoven P, et al. Serum levels of brain-derived neurotrophic factor in major depressive disorder: state-trait issues, clinical features and pharmacological treatment. Mol Psychiatry 2011; 16: 1088-95.

39 Goodyer IM. Genes, environments and depressions in young people. Arch Dis Child 2015; published online April 15. DOI:10.1136 archdischild-2014-306936.

40 Robinson OJ, Sahakian BJ. Recurrence in major depressive disorder: a neurocognitive perspective. Psychol Med 2008; 38: 315-18.

41 Roth TL, Lubin FD, Funk AJ, Sweatt JD. Lasting epigenetic influence of early-life adversity on the BDNF gene. Biol Psychiatry 2009; 65: 760-69.

42 Roiser JP, Elliott R, Sahakian BJ. Cognitive mechanisms of treatment in depression. Neuropsychopharmacology 2012; 37: 117-36. 